\title{
The Marginal Effects in Subgroup Decomposition of the Gini Index
}

\author{
Tomson Ogwang
}

\begin{abstract}
In this article, we derive the elasticity of the Gini index with respect to changes in subgroup incomes for subgroups that are characterized by significant income separation. The resulting elasticity, which is structurally similar to that of the empirically popular Lerman and Yitzhaki's (1985) elasticity for Gini income-source decomposition, entails easy and transparent computations. Some possible checks for income separation are described and an illustrative example using Canadian data is provided. The advantages of the proposed methodology over the Shapley value approach to Gini subgroup decomposition are stated.
\end{abstract}

Key words: Subgroup decomposition; income separation; Gini index; elasticity; pseudoLorenz regression curve.

\section{Introduction}

In a recent article, Ogwang (2014) developed a convenient method of decomposing the Gini index by population subgroups where the subgroups may be formed by gender, race, occupation, region, and so on. The advantage of Ogwang's approach is that the overall Gini index is simultaneously decomposed into the traditional within-group, between-group, and interaction (overlapping) components as well as decomposed by the contributions of the various subgroups to overall inequality. Hence, Ogwang's approach entails a two-way decomposition of the Gini index solely by population subgroups.

Ogwang's approach is able to isolate the contributions of the various subgroups to the overall Gini index, providing an alternative way of reconciling Gini subgroup decomposition with its income-source decomposition, for which the contributions of the various income sources to the overall Gini index are also isolated. It should be mentioned from the outset that other ways of reconciling subgroup decomposition of the Gini index with its income-source decomposition have also been proposed in the literature. One example of such a reconciliation is the multidimensional decomposition considered by Mussard (2004), Mussard and Richard (2012), Mussard and Savard (2012), and Mussini (2013), among others, which entails simultaneous decomposition of the Gini index by

\footnotetext{
${ }^{1}$ Brock University - Economics, 500 Glenridge Avenue St. Catharines Ontario L2S3A1, Canada. Email: togwang@brocku.ca

Acknowledgments: The author would like to thank D. Cho, L. Kwong, J. F. Lamarche, R. Watuwa, the Associate Editor and three anonymous referees for their valuable comments. Thanks are also extended to the seminar participants at the African Economic Research Consortium in Nairobi for their valuable insights. Research support from the Council for Research in the Social Sciences at Brock University is also gratefully acknowledged. The usual disclaimer is applicable.
} 
population subgroups and income source. Other examples include the regression approach to inequality decompositions (e.g., Cowell and Fiorio 2011) and the Shapley decomposition (e.g., Chantreuil and Trannoy 2011; Shorrocks 2013).

From a policy perspective, the information on the contributions of the various subgroups to the overall Gini index is potentially very useful. This is because the contributions could in principle be used in the analysis of the marginal effects by revealing the elasticity of the Gini index with respect to subgroup-income changes. The elasticity statistic, which indicates the extent to which a small proportionate change in the incomes of the members of a particular population subgroup increases or decreases overall inequality, controlling for the incomes of the members of other population subgroups, aids policy makers in devising appropriate inequality-reduction strategies. For example, the statistic could be used to gauge the extent to which income changes (e.g., transfer payments) targeting members of a particular population subgroup (e.g., race) affect the overall Gini index. Hence, it is important to report the relevant elasticities in empirical studies involving subgroup decompositions of the Gini index.

One of the earliest endeavors to derive the elasticity of the Gini index with respect to changes in subgroup incomes as a function of the constituent subgroup concentration indexes was proposed by Podder (1993). Subsequently, Aaberge et al. (2005) developed a general theoretical framework for determining the elasticity of the Gini index with respect to changes in subgroup incomes from the estimated parameters of the so-called pseudoLorenz regression curve. Mussard and Richard (2012) and Jurkatis and Strehl (2014) have considered the marginal effects, including elasticities, in the context of multidimensional decompositions of the Gini index.

Shorrocks (2013) has articulated how the Shapley decomposition could be used in the analysis of the marginal effects in both subgroup and income decompositions of any inequality measure, including the Gini index. In principle, the Shapley decomposition could be extended to the elasticity analysis. Charpentier and Mussard (2011), Cowell and Fiorio (2011), Chantreuil and Trannoy (2011), and Shorrocks (2013), among others, provide detailed discussions of the strengths and weaknesses of the Shapley decomposition. One of the main strengths of the decomposition, which is relevant for elasticity analysis, is the ability to express overall inequality (or poverty) as the sum of the contributory factors. Three major weaknesses of the Shapley decomposition in the context of the Gini index are particularly noteworthy. First, as pointed out in Section 3 below, the Shapley subgroup decomposition of the Gini index entails loss of information contained in the interaction component of the Gini index. This is because in the decomposition the interaction component of the Gini index is absorbed into the within-group or between-group component. Second, as pointed out by Shorrocks $(2013,117)$, the Shapley subgroup decomposition does not solve the socalled "subgroup inconsistency" problem associated with the Gini index, where subgroup inconsistency refers to a situation where overall inequality (as measured by the Gini index) could rise even though inequality in every constituent subgroup has fallen and the subgroup mean incomes and subgroup sizes are unchanged. Third, the Shapley decomposition results are sensitive to the structure of the income inequality game (Charpentier and Mussard 2011) or the hierarchical structures associated with the game (Chantreuil and Trannoy 2011). 
Although many empirical papers that have reported the elasticity of the Gini index in the context of income-source decompositions can be found in the literature (e.g., Lerman and Yitzhaki 1985; Stark et al. 1986; Leibbrandt et al. 2000; Lopéz-Feldman 2006), it is not yet commonplace for empirical researchers to report the elasticity in the context of Gini subgroup decompositions - even though this elasticity has policy significance, as has already been alluded to above. Podder (1993) and Chatterjee and Podder (2007) are rare empirical papers reporting the elasticity of the Gini index with respect to changes in subgroup incomes. The elasticities they report are functions of the concentration indexes for the constituent population subgroups. An extensive literature search reveals hardly any empirical papers that report elasticities in the context of the Shapley value approach to Gini subgroup decomposition.

The paucity of empirical papers reporting the elasticity of the Gini index with respect to changes in subgroup incomes is partly ascribed to the difficulties with the empirical implementation of the existing methods. Hence, the search for convenient methods of estimating the elasticity continues.

An important first step in the derivation of the Gini subgroup decomposition elasticities is to isolate the contributions of the various subgroups to the overall Gini index. Hence, in principle, any approach to Gini subgroup decomposition that isolates the contributions of the various subgroups to the overall Gini index could be exploited in the derivations of the relevant elasticities, albeit with varying degrees of difficulty. For example, as pointed out by a referee, the subgroup decomposition considered by Radaelli (2010), which entails a breakdown of the overall Gini index into a withingroup component and a between-group component in two steps, provides the contributions of the various subgroups to the overall Gini index. However, this approach, like the Shapley decomposition, cannot be conveniently extended for purposes of elasticity analysis.

As already mentioned above, the approach developed by Ogwang (2014) also enables the isolation of the contributions of the various subgroups to the overall Gini index. In light of the computational convenience associated with Ogwang's approach, it makes sense to exploit the approach for the purposes of Gini elasticity analysis.

Therefore, the present article aims to extend Ogwang's 2014 article by deriving the elasticity of the Gini index with respect to changes in the incomes of all the incomereceiving units constituting a particular population subgroup. The derivation is made under the assumption of significant subgroup-income separation. Under this assumption, small proportionate changes in the incomes of all income-receiving units in the subgroup of interest do not change the rankings of these income-receiving units relative to those of all receiving units in all population subgroups; that is, the new ranks after the income changes are identical to the corresponding ranks before the changes. As will be seen below, the resulting elasticity turns out to be structurally similar to that of the widely reported Lerman and Yitzhaki's (1985) elasticity for Gini income-source decompositions. Some plausible empirical situations that entail subgroup-income separation are mentioned below, as are possible income-separation checks.

The rest of the article is structured as follows: in Section 2, we derive the elasticity of the Gini index with respect to changes in subgroup incomes under the assumption of significant subgroup-income separation. In Section 3, some possible checks for income 
separation are briefly described. An illustrative example using Canadian data is provided in Section 4. The concluding remarks are made in Section 5.

\section{Deriving the Gini Subgroup Decomposition Elasticities}

To facilitate the exposition of the Gini subgroup decomposition elasticities, it is helpful to provide a brief overview of the nature of the contributions of the subgroups to the overall Gini index, as presented in Ogwang's (2014) article, from which the marginal effects are then derived in this article.

Suppose that $n$ income-receiving units are classified into $k$ mutually exclusive and exhaustive subgroups (e.g., by gender, age, race, education, occupation, or region). The $k$ subgroups are arranged in ascending order of their subgroup mean incomes, but it is not necessary to arrange the incomes within each subgroup in any particular order.

We shall adopt the following notations also employed in Ogwang's (2014) article:

$n_{j}(j=1,2, \ldots, k)$ : the number of income-receiving units in the subgroup with the $j$ th smallest mean income.

$n=\sum_{j=1}^{k} n_{j}$ : the total number of income-receiving units in all the $k$ subgroups.

$y_{i j}\left(i=1,2, \ldots, n_{j} ; j=1,2, \ldots, k\right)$ : the income of the $i$ th income-receiving unit in the subgroup with the $j$ th smallest mean income in which case $\bar{y}_{j}=\left(1 / n_{j}\right) \sum_{i=1}^{n_{j}} y_{i j}$ is the mean income for the same subgroup.

$r_{i j}\left(i=1,2, \ldots, n_{j} ; j=1,2, \ldots, k\right)$ : the rank of $y_{i j}$ in relation to the incomes of all the $n=\sum_{j=1}^{k} n_{j}$ income-receiving units in all the $k$ subgroups.

$r_{i j}^{\prime}\left(i=1,2, \ldots, n_{j} ; j=1,2, \ldots, k\right)$ : the rank of $y_{i j}$ in relation to the incomes of only the $n_{j}$ income-receiving units in the subgroup with the $j$ th smallest mean income $\tilde{r}_{i j}\left(i=1,2, \ldots, n_{j} ; j=1,2, \ldots, k\right)$ : the rank of $y_{i j}$ in relation to the incomes of all the $n=\sum_{j=1}^{k} n_{j}$ income-receiving units, assuming that $y_{i j}=\bar{y}_{j}$.

In the construction of the ranks $r_{i j}, r_{i j}^{\prime}$, and $\tilde{r}_{i j}$ tied incomes are assigned the average of the ranks they would have been assigned assuming that they were not tied. For example, if under a particular ranking rule two incomes are tied and one of them would have been assigned a rank of two and the other would have been assigned a rank of three in the absence of ties, then under this ranking rule both incomes are assigned the average rank of $(2+3) / 2=2.5$.

The following rank transformations are also required: $r_{i j}^{*}=2 r_{i j}-n-1$ $\left(i=1,2, \ldots, n_{j} ; j=1,2, \ldots, k\right), r_{i j}^{* \prime}=2 r_{i j}^{\prime}-n_{j}-1\left(i=1,2, \ldots, n_{j} ; j=1,2, \ldots, k\right)$; $\tilde{r}_{i j}^{*}=2 \tilde{r}_{i j}-n-1\left(i=1,2, \ldots, n_{j} ; j=1,2, \ldots, k\right) ; \widehat{r}_{i 1}^{*}=\left(r_{i 1}-r_{i 1}{ }^{\prime}\right), i=1,2, \ldots, n_{1}$ and $\hat{r}_{i j}^{*}=\left(r_{i j}-r_{i j}^{\prime}-\sum_{i=1}^{j-1} n_{i}\right), i=1,2, \ldots, n_{j} ; j=2, \ldots, k$.

Following Ogwang (2014), the overall Gini index is given by

$$
G=\sum_{j=1}^{k} p_{j} s_{j} G_{W j}+\sum_{j=1}^{k} p_{j} s_{j} G_{B j}+2 \sum_{j=1}^{k} p_{j} s_{j} G_{I j}
$$

where $p_{j}=n_{j} / n$ is the population share of the subgroup with the $j$ th smallest mean income, $j=1,2, \ldots, k ; s_{j}=\left(\sum_{i=1}^{n_{j}} y_{i j}\right) / \sum_{i=1}^{n_{j}} \sum_{j=1}^{k} y_{i j}$ is the income share of the subgroup with the $j$ th smallest mean income, $j=1,2, \ldots, k$; and $G_{W j}, G_{B j}$, and $G_{I j}$ are the within-group Gini, the between-group pseudo-Gini, and the interaction pseudo-Gini, respectively, for the subgroup with the $j$ th smallest mean income, $j=1,2, \ldots, k$. The relevant formulas 
for computing $G_{W j}, G_{B j}$, and $G_{I j}$ are as follows:

$$
\begin{gathered}
G_{W j}=\frac{1}{n_{j}} \frac{\sum_{i=1}^{n_{j}} r_{i j}^{* \prime} y_{i j}}{\sum_{i=1}^{n_{j}} y_{i j}} \\
G_{B j}=\frac{1}{n_{j}} \frac{\sum_{i=1}^{n_{j}} \tilde{r}_{i j}^{*} y_{i j}}{\sum_{i=1}^{n_{j}} y_{i j}} \\
G_{I j}=\frac{1}{n_{j}} \frac{\sum_{i=1}^{n_{j}} \hat{r}_{i j}^{*} y_{i j}}{\sum_{i=1}^{n_{j}} y_{i j}}
\end{gathered}
$$

Ogwang articulates how $G_{W j}, G_{B j}$, and $G_{I j}$ can be conveniently estimated by setting up artificial regressions with known heteroscedastic structures for which the variance of the error term is related to the incomes of the income-receiving units in the subgroup with the jth smallest mean income.

The first, second, and third terms on the right-hand side of Equation (1) are the withingroup, between-group, and interaction components, respectively. It is also apparent from the same equation that the contribution of the subgroup with the $j$ th smallest mean income to the overall Gini index is given by

$$
G_{j}=p_{j} s_{j} G_{W j}+p_{j} s_{j} G_{B j}+2 p_{j} s_{j} G_{I j}
$$

The first, second, and third terms on the right-hand side of Equation (5) represent the subgroup's contribution to the within-group, between-group, and interaction components, respectively, of the overall Gini index. As will be discussed in Section 3 below, the third term on the right-hand side of Equation (5), which defines the contribution of the subgroup with the $j$ th smallest mean income to the interaction component, is a possible measure of the extent to which the incomes of the income-receiving units in this subgroup are separated from those of the income-receiving units in all other subgroups.

We now turn our attention to the effect of increasing the incomes of all the income-receiving units in the subgroup with the $j$ th smallest mean income by a small proportion $\delta>0$. Potentially, this income increase could change the rankings of the income-receiving units in this subgroup relative to those of the income-receiving units in all the subgroups, thereby complicating the analysis. To circumvent this problem, it is necessary to invoke the assumption of income separation. As suggested by a referee, the income-separation assumption may be called the "rank-preserving condition." Hence, hereafter, the terms "incomeseparation assumption" and "rank-preserving condition" will be used interchangeably.

Given that income separation is an important assumption in the analysis of the marginal effects, it is pertinent to elaborate on what income separation entails. Strictly speaking, income separation refers to situations for which the incomes of the members of a particular subgroup are removed from those of the members of other population subgroups. Under such a separation, small proportionate changes in the incomes of all income-receiving units in the separated population subgroup do not change the rankings of these incomereceiving units relative to those of the income-receiving units in all population subgroups. 
In general, income separation applies to subgroup formations with some nonoverlapping income ranges. One such formation, which commonly features in official statistics releases, is by income quantiles. This subgroup formation results in the interaction component of the overall Gini index being zero. Other subgroup formations, such as poor/rich and low income/high income, also give rise to nonoverlapping income ranges. The elasticity derived in this article applies to these subgroup formations, provided that small proportionate changes in the incomes of the income-receiving units in the first subgroup do not result in some of its members becoming richer than the income-receiving units in the second subgroup.

In some empirically likely situations, income separation may only apply to a few subgroups whose incomes are far removed from those of the other subgroups. In these situations, it is appropriate to analyze the marginal effects only for the subgroup(s) whose incomes are deemed to be separated from those of other subgroups. The issue of checking for subgroup-income separation is discussed in the next section.

In fact, owing to the complications arising from the existence of an interaction component, multidimensional decompositions of the Gini index have traditionally focused on the case of subgroups with nonoverlapping income ranges (e.g., Mussard and Richard 2012). Also, the concentration-ratio-based elasticities reported by Podder (1993) and Chatterjee and Podder (2007) are strictly valid under the assumption of significant subgroup-income separation although these researchers justified their reporting of these elasticities based on the assumption that the proportional income changes are too small to have any effect on the relative ranks.

Let $G(j, 0)$ and $G(j, \delta)$ denote the Gini index before increasing and after increasing, respectively, the incomes of the members of the subgroup with the $j$ th smallest mean income by the proportion $\delta$. It is easy to verify that under income separation, the mean income for the subgroup is increased by the same proportion $\delta$ but the within-group Ginis $\left(G_{W j}, j=1,2, \ldots, k\right)$, the between-group pseudo-Ginis $\left(G_{B j}, j=1,2, \ldots, k\right)$, and the interaction pseudo-Ginis $\left(G_{I j}, j=1,2, \ldots, k\right)$ are unchanged. However, the income share for the subgroup with the $j$ th smallest mean income increases from $s_{j}=\frac{\sum_{i=1}^{n_{j}} y_{i j}}{\sum_{i=1}^{n_{j}} \sum_{j=1}^{k} y_{i j}}$ to $s_{j}+B_{j}$ where $B_{j}=\frac{\delta \sum_{i=1}^{n_{j}} y_{i j} \sum_{i=1}^{n_{j}} \sum_{j=1}^{k} y_{i j}-\delta\left(\sum_{i=1}^{n_{j}} y_{i j}\right)^{2}}{\left(\sum_{i=1}^{n_{j}} \sum_{j=1}^{k} y_{i j}\right)^{2}+\delta \sum_{i=1}^{n_{j}} \sum_{j=1}^{k} y_{i j} \sum_{i=1}^{n_{j}} y_{i j}}$ whereas the income share for the subgroup with the $r$ th smallest mean income, where $r \neq j$, decreases from $s_{r}=\frac{\sum_{i=1}^{n_{r}} y_{i r}}{\sum_{i=1}^{n_{j}} \sum_{j=1}^{k} y_{i j}}$ to $s_{r}+A_{r}$ where $A_{r}=\frac{-\delta \sum_{i=1}^{n_{r}} y_{i r} \sum_{i=1}^{n_{j}} y_{i j}}{\left(\sum_{i=1}^{n_{j}} \sum_{j=1}^{k} y_{i j}\right)^{2}+\delta \sum_{i=1}^{n_{j}} \sum_{j=1}^{k} y_{i j} \sum_{i=1}^{n_{j}} y_{i j}}$.

Substituting the new income shares into Equation (1) taking into account the fact that the within-group Ginis, the between-group pseudo-Ginis and the interaction pseudo-Ginis are unchanged under the separability assumption, as already alluded to above, as well as the fact that $\lim (\delta \rightarrow 0)\left(B_{j} / \delta\right)=s_{j}\left(1-s_{j}\right)$ and $\lim (\delta \rightarrow 0)\left(A_{r} / \delta\right)=-s_{r} s_{j}$, it follows that

$$
\begin{aligned}
{[G(j, 0)]^{-1} \lim (\delta} & \rightarrow 0)\left(\frac{G(j, \delta)-G(j, 0)}{\delta}\right) \\
& =[G(j, 0)]^{-1}\left[G_{j}-\sum_{i=1}^{k} s_{j} G_{i}\right]=[G(j, 0)]^{-1}\left[G_{j}-s_{j} \sum_{i=1}^{k} G_{i}\right]
\end{aligned}
$$


Since $\sum_{i=1}^{k} G_{i}=G(j, 0)$, i.e., the sum of the contributions of the $k$ population subgroups is equal to the overall Gini index, it follows that the elasticity of the Gini index with respect to changes in the incomes of all income-receiving units in the subgroup with the $j$ th smallest mean income component is given by

$$
\eta_{j}=\left[\left\{G_{j} / G(j, 0)\right\}-s_{j}\right]
$$

To the best of our knowledge, the elasticity formula given by Equation (7), which is clearly very simple, has not previously been featured in the literature on subgroup decomposition of the Gini index (detailed derivations of the results are available from the author).

Five important features of Equation (7) are worthy of mention. First, the elasticity is structurally similar to that of the empirically popular Lerman and Yitzhaki's (1985) elasticity for Gini income-source decomposition. Specifically, the elasticity of the Gini index with respect to small proportional changes in subgroup incomes is the difference between the ratio of the contribution of the subgroup to the overall Gini index prior to the income change (i.e., $G_{j} / G(j, 0)$ ) and the income share of that subgroup prior to the change (i.e., $s_{j}$ ). Likewise, Lerman and Yitzhaki's elasticity with respect to small proportional changes in the incomes of all receiving units from a particular source is the difference between the ratio of the contribution of that income source to the overall Gini index prior to the income change and the share of that income source in total income prior to the change. Second, given that the ranks are unchanged under subgroup-income separability, the resulting elasticity given by Equation (7) solely reflects the share effects. Third, as the illustrative example presented in Section 4 below also indicates, the elasticity could be positive, zero, or negative depending on the magnitude of $G_{j}$, since $G(j, 0)$ and $s_{j}$ cannot be negative. Fourth, $\sum_{j=1}^{k} \eta_{j}=0$. The zero sum of all the elasticities is an artifact of the scale-independence property of the Gini index, which guarantees that increasing the incomes of all the receiving units in all population subgroups by a constant proportion does not affect the overall Gini index. Fifth, the computations entailed are transparent and straightforward.

\section{Checking for Income Separation}

It is repeatedly stressed in this article that income separation is necessary for the elasticity formula as given by Equation (7) to be strictly valid. Hence, it is important to check each subgroup for income separation to determine whether it is appropriate to compute the elasticity for that subgroup. In cases where the subgroups are formed by income quantiles, as is the case with the illustrative example presented in Section 4 below, the elasticities can be reported for all quantiles since all of them would satisfy income separation.

In other cases, however, it is necessary to check individual subgroups for income separation and only report the elasticities for those subgroups that are deemed to satisfy the income-separation (or rank-preserving) condition. In this regard, the contribution of the interaction component to the overall Gini index, as defined by the third term on the righthand side of Equation (5), provides a simple check of how segregated the incomes of the income-receiving units in this subgroup are from those of the income-receiving units of all other subgroups. Specifically, if the incomes in the subgroup with the $j$ th smallest mean 
income are characterized by significant income separation, then the third term on the right-hand side of Equation (5) should be (very close to) zero. One positive aspect of this simple income-separation check is that subgroup size, an important consideration in the development of appropriate segregation indexes, is automatically taken into account via the population shares. Clearly, the contribution of each subgroup to the interaction component for the overall Gini index is beneficial in that it provides a simple but valid check for income separation.

Yitzhaki and Lerman (1991) proposed a stratification index that indicates the extent to which the incomes in a particular subgroup are separated from those in all other subgroups. Further details about Yitzhaki and Lerman's stratification index can be found in their paper. Yitzhaki (1994) and Allanson (2014), among others, propose indexes of overall income stratification that provide useful insights into the overall extent of income separation.

Regardless of which method is used to check for income separation, the elasticities should be reported only for the individual subgroups that are deemed to satisfy the appropriate income-separation (or rank-preserving) condition.

Before presenting an illustrative example using Canadian data, it is important to state the advantages of the marginal analysis of the Gini subgroup decompositions as developed in this article over the marginal analysis based on the Shapley decomposition. First, the fact that the Shapley approach results in the interaction term for the Gini index being absorbed into the within-group or the between-group component results in loss of information on the contribution of the subgroups to the interaction component of the Gini index, information which could be used to measure income separation as described in this section. Second, from a practical perspective the elasticities developed in this article are computationally more convenient, involving simple analytical formulas as opposed to the complex algorithms entailed in the Shapley decompositions.

\section{Illustrative Example Using Canadian Data}

To demonstrate the computation and interpretation of the elasticity of the Gini index with respect to changes in subgroup incomes, we applied the methodology described in Section 2 to the data on the total pretax post-transfer incomes, in Canadian dollars, of a random sample of 4,883 persons, derived from the Canadian Census 2006 Public Use Microdata Files. The sample data are available from the author upon request. To ensure that the appropriate conditions for the empirical validity of the elasticity are met, we first created nonoverlapping subgroups by categorizing the incomes into quintiles.

As indicated above, an important first step in the computation of the elasticities of the Gini index with respect to changes in subgroup incomes is the computation of the contributions of the various subgroups to the overall Gini index. These contributions are reported in the second last column of Table 1. The corresponding elasticities, which are computed using Equation (7), are reported in the last column of the same table.

It is apparent from the entries in the last column of Table 1 that the contribution of the income-receiving units in each of the first two quintiles to the overall Gini index is negative, whereas the contributions of those in the other quintiles are positive. These results raise the issue of the conditions under which the contributions of a particular 


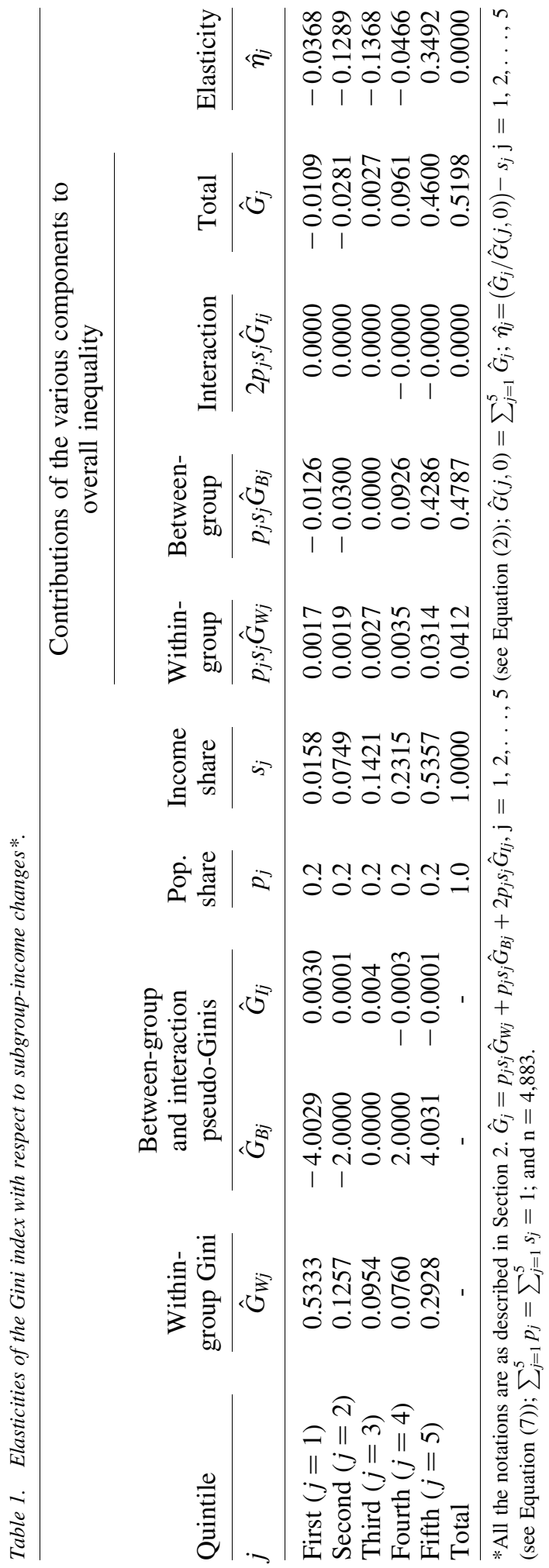


subgroup could be negative. To see how the contributions to the overall Gini index of the income-receiving units in the lower quintiles could be negative, we refer to Ogwang's (2014) analytical results, which indicate that the within-group Ginis are always positive whereas the between-group and interaction pseudo-Ginis can be positive or negative. Since the income shares and population shares cannot be negative, it follows from Equation (5) that the net contribution of a particular quintile could be positive or negative. In the present empirical example, it turns out that for the first two quintiles the negative effects dominate the positive effects associated with the within-group component. Podder (1993) and Chatterjee and Podder (2007) also uncovered negative contributions of some population subgroups to the overall Gini index when they applied the concentration-indexbased measures to their datasets.

Our experience with this dataset and several other datasets reveals that when most of the incomes in a particular subgroup fall below the median income, then the between-group pseudo-Gini associated with that subgroup will be negative. In fact, the corresponding concentration index for this subgroup formation also turns out to be negative, as pointed out by Chatterjee and Podder (2007).

As can be expected from the traditional subgroup decompositions of the Gini index, the overall Gini index and its within-group, between-group, and interaction components are all non-negative. It also turns out that the between-group inequality accounts for the largest proportion of the overall inequality and the interaction component accounts for the least proportion.

The entries in the third from last column of Table 1 also indicate that the sum of the contributions of the five quintiles to the interaction component is zero. This zero sum of the contributions to the interaction component can be expected, given that quintile formation ensures that there is no income overlapping.

The elasticities for the first four quintiles, reported in the last column of Table 1, are negative, whereas that for the top quintile is positive. The reported elasticities indicate that a one-percent increase in the incomes of the income-receiving units in the first, second, third, and fourth quintile, controlling for the incomes of the income-receiving units in all other quintiles in each case, will decrease the overall Gini index by 0.0368 percent, 0.1289 percent, 0.1368 percent, and 0.0466 percent, respectively. Also, a one-percent increase in the incomes of the income-receiving units in the top quintile, controlling for the incomes of the income-receiving units in all other quintiles, will increase the overall Gini index by 0.3492 percent. As anticipated, the sum of the elasticities for all the five quintiles is zero.

It is apparent from the results for the third and fourth quintiles that although a particular subgroup may initially make a positive contribution to the overall Gini index, small proportionate changes in the incomes of the members of this subgroup may result in a decrease in overall income inequality. This raises the interesting issue of whether empirical researchers should be focusing more on the contributions of the various subgroups to overall inequality or on the elasticities. With respect to this issue, Jurkatis and Strehl (2014) argue in favor of focusing more strongly on the elasticities, given the policy significance. The fact that the elasticities for the lower-income quintiles are negative and that for the top-income quintile is positive should not be surprising, given that increasing the incomes of the income-receiving units in a lower quintile, controlling for 
other incomes, would potentially lead to a closure of the income gap. However, in general empirical situations with subgroup-income interactions, the link between proportionate marginal subgroup-income changes and overall income inequality is convoluted.

\section{Concluding Remarks}

The elasticity of the Gini index with respect to changes in subgroup incomes is a statistic that aids policy makers in devising appropriate inequality-reduction strategies. Presenting data on the elasticities may also help the various stakeholders to gain valuable insights from the decomposition.

The trick in deriving this elasticity is to first isolate the contributions of the various subgroups to the overall Gini index from which the marginal effects are then established. Since the marginal income changes could potentially result in relative rank changes, which complicate the analysis, it becomes necessary to invoke the rank-preserving condition. This condition applies if there is a high degree of income separation in that the incomes of the members comprising of a particular subgroup are very different from the incomes of the members of other subgroups (e.g., they are too high or too low). Under income separation, it is apparent from Equation (7) that the elasticity is simply the difference between the ratio of the contribution of that subgroup to the overall Gini index prior to the income change and the income share of the same subgroup prior to the change.

It is also possible in principle to account for sampling variability in the elasticity statistic by computing its bootstrap or jackknife standard error, which is valuable for hypothesistesting purposes. In this regard, accuracy considerations necessitate the re-ranking of the incomes in each bootstrap/jackknife subsample in accordance with the ranking rules described in Section 2, which is computationally intensive but should not be overly difficult if the empirical researcher has access to an appropriate ranking subroutine. Shao and Tu (1995) explain the bootstrap and jackknife methodologies in general and Ogwang (2014) explains the calculation of jackknife standard errors in the subgroup decomposition of the Gini index.

It has been mentioned in the literature (e.g., Podder 1993) that the problem of the inability to neatly decompose the Gini index into only two components (i.e., within-group component and between-group component) owing to the existence of a third component, the interaction component, is circumvented by focusing on the contributions of the various subgroups to the overall Gini index. However, it is apparent from the discussion in this article that this problem in fact resurfaces in the analysis of the elasticities unless the subgroup under consideration is characterized by significant income separation.

Finally, we hope that the results presented in this article will help to popularize the reporting of the elasticities of the Gini index with respect to changes in subgroup incomes in empirical investigations involving population subgroups that are characterized by significant income separation.

\section{References}

Aaberge, R., S. Bjerve, and K. Doksum. 2005. "Decomposition of Rank-Dependent Measures of Inequality by Subgroups." Metron-International Journal of Statistics 
LXIII: 493-503. Available at: ftp://metron.sta.uniroma1.it/RePEc/articoli/si11.pdf. (accessed June 30, 2015).

Allanson, P. 2014. "Income Stratification and Between-group Inequality." Economics Letters 124: 227-230. Doi: http://dx.doi.org/10.1016/j.econlet.2014.05.025.

Chantreuil, F. and A. Trannoy. 2011. "Inequality Decomposition Values." Annals of Economics and Statistics 101/102: 13-36. Available at: http://www.jstor.org/stable/ 41615472 (accessed June 30, 2015).

Charpentier, A. and S. Mussard. 2011. "Income Inequality Games.” Journal of Economic Inequality 9: 529-554. Doi: http://dx.doi.org/10.1007/s10888-011-9184-1.

Chatterjee, S. and N. Podder. 2007. "Some Ethnic Dimensions of Income Distribution from Pre- to Post-Reform New Zealand, 1984-1998." The Economic Record 83: 275-287. Doi: http://dx.doi.org/10.1111/j.1475-4932.2007.00414.x.

Cowell, F.A. and C.V. Fiorio. 2011. "Inequality Decompositions - A Reconciliation." Journal of Economic Inequality 9: 509-528. Doi: http://dx.doi.org/10.1007/ s10888-011-9176-1.

Jurkatis, S. and W. Strehl. 2014. "Gini Decompositions and Gini Elasticities: On Measuring the Importance of Income Sources and Population Subgroups for Income Inequality." Discussion Papers 2014/22, Freie Universität Berlin, School of Business and Economics. Available at: http://econstor.eu/bitstream/10419/102730/1/798627875.pdf (accessed June 30, 2015).

Leibbrandt, M., C. Woolard, and I. Woolard. 2000. "The Contribution of Income Components to Income Inequality in the Rural Former Homelands of South Africa: A Decomposable Gini Analysis.” Journal of African Economies 9: 77-99. Doi: http://dx. doi.org/10.1093/jae/9.1.79.

Lerman, R.I. and S. Yitzhaki. 1985. "Income Inequality Effects by Income Source: A New Approach and Applications to the United States." Review of Economics and Statistics 67: 151-156. Doi: http://dx.doi.org/10.2307/1928447.

Lopéz-Feldman, A. 2006. "Decomposing Inequality and Obtaining Marginal Effects." The Stata Journal 6: 106-111. Available at: http://www.stata-journal.com/article. html? article $=$ st0100 (accessed June 30, 2015).

Mussard, S. 2004. "The Bidimensional Decomposition of the Gini Ratio. A Case Study: Italy." Applied Economics Letters 11: 503-505. Doi: http://dx.doi.org/10.1080/ 1350485042000244530.

Mussard, S. and P. Richard. 2012. "Linking Yitzhaki's and Dagum's Gini Decompositions." Applied Economics 44: 2997-3010. Doi: http://dx.doi.org/10.1080/ 00036846.2011 .568410$.

Mussard, S. and L. Savard. 2012. "The Gini Multi-Decomposition and the Role of Gini's Transvariation: Application to Partial Trade Liberalization in the Philippines." Applied Economics 44: 1235-1249. Doi: http://dx.doi.org/10.1080/00036846.2010.539540.

Mussini, M. 2013. "A Matrix Approach to the Gini Index Decomposition by Subgroup and Income Source." Applied Economics 13: 2457-2468. Doi: http://dx.doi.org/10.1080/ 00036846.2012 .667553$.

Ogwang, T. 2014. "A Convenient Method of Decomposing the Gini Index by Population Subgroups.” Journal of Official Statistics 30: 91-105. Doi: http://dx.doi.org/10.2478/ jos-2014-0005. 
Podder, N. 1993. "A New Decomposition of the Gini Coefficient among Groups and its Interpretations with Applications to Australia." Sankhya: The Indian Journal of Statistics 55: 262-271. Available at: http://www.jstor.org/stable/25052790 (accessed June 30, 2015).

Radaelli, P. 2010. "On the Decomposition by Subgroups of the Gini Index and Zenga's Uniformity and Inequality Indexes." International Statistical Review 78: 81-101. Doi: http://dx.doi.org/10.1111/j.1751-5823.2010.00100.x.

Shao, J. and D. Tu. 1995. Jackknife and Bootstrap. New York: Springer.

Shorrocks, A.F. 2013. "Decomposition Procedures for Distributional Analysis: A Unified Framework Based on the Shapley Value." Journal of Economic Inequality 11: 99-126. Doi: http://dx.doi.org/10.1007/s10888-011-9214-z.

Stark, O., J.E. Taylor, and S. Yitzhaki. 1986. "Remittances and Inequality." Economic Journal 96: 722-740. Available at: http://www.jstor.org/stable/2232987 (accessed June 30, 2015).

Yitzhaki, S. 1994. "Economic Distance and Overlapping of Distributions." Journal of Econometrics 61: 147-159. Doi: http://dx.doi.org/10.1016/0304-4076(94)90081-7.

Yitzhaki, S. and R.I. Lerman. 1991. "Income Stratification and Income Inequality." Review of Income and Wealth 37: 313-329. Doi: http://dx.doi.org/10.1111/ j.1475-4991.1991.tb00374.x.

Received February 2015

Revised July 2015

Accepted September 2015 